\title{
Proliferation of human hepatocellular carcinoma cells from surgically resected specimens under conditionally reprogrammed culture
}

\author{
ZHENGLU WANG ${ }^{1,2}$, BOWEN BI ${ }^{2}$, HONGLI SONG ${ }^{3}$, LEI LIU ${ }^{4}$, \\ HONG ZHENG ${ }^{5}$, SHUSEN WANG ${ }^{6}$ and ZHONGYANG SHEN $^{3,7}$
}

\author{
${ }^{1}$ Pathology Department; ${ }^{2}$ Biological Sample Resource Sharing Center; ${ }^{3}$ Organ Transplantation Department, \\ Tianjin First Center Hospital; ${ }^{4}$ Key Laboratory for Critical Care Medicine of the Ministry of Health; \\ ${ }^{5}$ Key Laboratory of Transplant Medicine, Chinese Academy of Medical Sciences; ${ }^{6}$ Tianjin Key Laboratory for \\ Organ Transplantation; ${ }^{7}$ Tianjin Clinical Research Center for Organ Transplantation, Tianjin 300192, P.R. China
}

Received July 3, 2018; Accepted March 25, 2019

DOI: $10.3892 / \mathrm{mmr} .2019 .10160$

\begin{abstract}
Hepatocellular carcinoma (HCC) is the third most common cause of cancer mortality worldwide, which is partially due to the lack of appropriate therapeutic options. The development of HCC is accompanied with unique and continuous genomic and epigenetic modifications. Therefore, the absence of a personalized and reproducible human model reduces the ability to determine the potential of candidate treatments. Conditional reprogramming (CR) culture has been used to establish and indefinitely grow patient-derived tumor cell lines in a rapid and efficient manner. In the present study, primary HCC cells were isolated from tumor specimens and cultured under CR conditions. The proliferative potential and capacity of cells to undergo continuous regeneration were evaluated by cell viability and proliferation assays, and the expression of tumor-specific markers was determined by western blotting and immunofluorescence to determine the prospects for use in clinical settings. It was demonstrated that $\sim 55 \%$ of tumor samples were able to generate HCC cells that could be continuously expanded and passaged under CR conditions; this ability was associated with the source and composition of the tumor tissues. Furthermore, the expression of the tumor-specific marker $\alpha$-fetoprotein and the proliferative ability of cells were maintained following cycles of
\end{abstract}

Correspondence to: Dr Zhongyang Shen, Organ Transplantation Department, Tianjin First Center Hospital, 24 Fukang Road, Nankai, Tianjin 300192, P.R. China

E-mail: SZY17246@outlook.com

Abbreviations: HCC, hepatocellular carcinoma; CR, conditional reprogramming; DMEM/F12, Dulbecco's modified Eagle's medium/ Nutrient Mixture F-12 medium; AFP, $\alpha$-fetoproteins

Key words: HCC, conditional reprogramming, tumor specimens, precision therapy cryopreservation and resuscitation. In conclusion, with further optimization, the CR system may be a useful tool for the precise therapeutic treatment of patients with HCC.

\section{Introduction}

Primary liver cancer, predominantly comprising hepatocellular carcinoma (HCC), is reported to be the fifth most common cancer globally and the third most common cause of cancer-associated mortality (1). There is an increasing understanding of the molecular mechanisms that induce hepatocarcinogenesis, including chronic infections such as hepatitis $\mathrm{B}$ or $\mathrm{C}(\mathrm{HBV} / \mathrm{HCV})$, alcohol abuse and metabolic syndromes (2). Surgical resection followed by adjuvant drug therapy is the most common treatment in clinical settings (3); however, the metastasis and chemoresistance of tumor cells results in poor outcomes for patients with advanced HCC (4). As the development of $\mathrm{HCC}$ is accompanied with unique and continuous genomic and epigenetic alterations, combining personalized approaches, including molecular analysis-guided targeted therapy and immunotherapy may be a potential strategy to improve the treatment of cancer $(5,6)$. Existing preclinical models comprise genetically engineered mouse models and human tumor-derived cell lines; however, a reproducible human model is required to accurately reproduce the important characteristics of tumors in vivo and determine the effectiveness of candidate therapeutics. Commercial tumor cell lines have been extensively used in the investigation of therapeutic targets; however, the establishment of in vitro models that use tumor cells from individual patients may serve to improve the clinical relevance of in vitro studies (3).

Tumor cells have been associated with strong proliferative ability. This property is detrimental for the rapid expansion of cells derived from adult tumor tissues while retaining stable lineage commitment, particularly from liver tumors (7). Conditional reprogramming (CR) systems have previously been used to establish patient-derived cell lines from normal and tumor tissues that possess the ability to grow indefinitely in vitro without genetic manipulation $(8,9)$. 
Potential applications for the CR system in clinical settings have been investigated for breast $(10,11)$, lung (12) and prostate cancers $(13,14)$; however, it has been hypothesized that the CR system cannot be used to expand patient-derived metastatic lung cancer cells (15). In an in vitro study of cultured liver cancer cells, Broutier et al (16) successfully constructed a primary HCC organoid based on the CR system using a three-dimensional (3D) culture method. On the contrary, whether $\mathrm{CR}$ may serve as a reliable in vitro culture method to obtain matched tumor cells from patients with HCC remains unclear.

The aim of the present study was to establish a culture system with potential clinical applications that enabled the amplification of genetically stable cells. Primary tumor cells were isolated from tissue specimens from 20 patients with HCC and were cultured using the CR system. The proliferative potential and capacity of cells to undergo continuous regeneration, and the expression of tumor-specific markers were evaluated to determine the prospects for use in clinical settings. The study provided a primary investigation into culture systems for HCC cells in vitro, in preparation for future studies involving the establishment of a conditionally reprogrammed culture model for drug screening in the treatment of liver cancer drug screening.

\section{Materials and methods}

Cell isolation. A total of 20 samples of liver tumor tissue were obtained from patients (10 males and 10 females, aged 38-67 years old) undergoing orthotopic liver transplantation (OLT) or hepatectomy at Tianjin First Center Hospital (Tianjin, China) between January 2015 and December 2017. Tumors were graded using the American Joint Commission on Cancer 8th edition staging system for patients with HCC. The study was approved by the Tianjin First Central Hospital Clinical Research Ethics Committee (review no. 2016N057KY). Informed written consent was obtained from all patients. Procedures were conducted in accordance with the Declaration of Helsinki (17). Surgically resected liver tumor tissue was obtained from individuals with HCC who had no history of viral-mediated hepatitis. Half of the tissue was de-identified and supplied to lab personnel for tissue culture; remaining tissue was used for histological analysis. A $1 \mathrm{~cm}^{3}$ section of tumor tissue was collected and transferred into a sterile tube containing $1 \mathrm{x}$ cell protective fluid (Beijing Percans Oncology Research Co., Ltd., Beijing, China). The tissues were qualitatively divided into two types: Hard tissues (brittle and non-viscous tissues) and soft tissues (tissues with loose texture consisting of lumpy masses). Following five washes with PBS, the samples were minced into $\sim 2 \mathrm{~mm}^{3}$ sections and treated using a gentleMACS ${ }^{\text {TM }}$ Tissue Dissociator (Miltenyi Biotec GmbH, Bergisch Gladbach, Germany). The samples were digested into single cells by $1 \mathrm{x}$ collagenase/hyaluronidase solution (Thermo Fisher Scientific, Inc., Waltham, MA, USA) followed by $0.25 \%$ trypsin-EDTA (Sigma-Aldrich; Merck KGaA, Darmstadt, Germany) at $37^{\circ} \mathrm{C}$ for $2 \mathrm{~h}$. Cell suspensions were filtered using a MACS $^{\circledR}$ SmartStrainer (Miltenyi Biotec $\mathrm{GmbH}$ ). The primary cells were suspended in Dulbecco's modified Eagle's medium/Nutrient Mixture F-12 medium (DMEM/F12; Hyclone; GE Healthcare Life Sciences,
Logan, UT, USA) and then centrifuged at $300 \mathrm{x}$ g for $5 \mathrm{~min}$ at $37^{\circ} \mathrm{C}$. Primary $\mathrm{HCC}$ cells were further purified from stromal cells using a magnetic bead isolate system (Beijing Percans Oncology Research Co., Ltd.) according to the manufacturer's protocols (18).

$H \& E$ staining. The tumor tissues were fixed with $4 \%$ paraformaldehyde overnight at $4^{\circ} \mathrm{C}$ and embedded in paraffin. The samples in paraffin were cut into $3-\mu \mathrm{m}$ sections, dewaxed in xylene and rehydrated in a graded alcohol series. Sections were stained with hematoxylin for $10 \mathrm{~min}$ at room temperature, and rinsed with tap water for $10 \mathrm{~min}$. Differentiation was performed with differentiation buffer in the H\&E staining kit (Beyotime Institute of Technology, Shanghai, China) for $30 \mathrm{sec}$ at room temperature to the obtain clear structure. Following further washing in running tap water for $10 \mathrm{~min}$, the sections were stained with eosin for $2 \mathrm{~min}$ at room temperature, then dehydrated and mounted. Then, sections were imaged using an optical microscope (magnification, x40; Eclipse 80i; Nikon Corporation, Tokyo, Japan); three images/sample were acquired for analysis.

$C R$ culture. In the CR system, mouse embryonic fibroblast cells (NIH-3T3; China Center for Type Culture Collection, Wuhan, China) were irradiated at $30 \mathrm{~Gy}$ with gamma radiation to provide an irradiated fibroblast feeder layer. HCC cells were passaged in DMEM/F12 containing the following: $10 \%$ fetal bovine serum (Gibco; Thermo Fisher Scientific, Inc.); $10 \mu \mathrm{g} / \mathrm{ml} \mathrm{Y}-27632$ (Enzo Life Sciences, Inc., Farmingdale, NY, USA); $0.4 \mu \mathrm{g} / \mathrm{ml}$ hydrocortisone (Sigma-Aldrich; Merck $\mathrm{KGaA}$ ); $5 \mu \mathrm{g} / \mathrm{ml}$ insulin (Thermo Fisher Scientific, Inc.); $8.6 \mathrm{ng} / \mathrm{ml}$ cholera toxin (Sigma-Aldrich; Merck KGaA); $20 \mathrm{ng} / \mathrm{ml}$ epidermal growth factor (Sigma-Aldrich; Merck $\mathrm{KGaA}$ ), $20 \mathrm{ng} / \mathrm{ml}$ hepatocyte growth factor (Sigma-Aldrich; Merck KGaA), $24 \mu \mathrm{g} / \mathrm{ml}$ adenine (Sigma-Aldrich; Merck $\mathrm{KGaA}$ ) and $3 \mathrm{nM}$ dexamethasone (Sigma-Aldrich; Merck $\mathrm{KGaA}$ ). The feeder cells were removed following incubation for $1 \mathrm{~min}$ in $0.05 \%$ Trypsin-EDTA (Sigma-Aldrich; Merck $\mathrm{KGaA})$ at $37^{\circ} \mathrm{C}$ and replaced with fresh cells $\left(4 \times 10^{4}\right.$ cells $\left./ \mathrm{cm}^{2}\right)$ during the passage of HCC-CR cells. A 6-day culture period was required to generate larger cell clones prior to the initial passage of P0 cells, then the cells were passaged every 3-4 days. The HCC-CR cells were sub-cultured at a 1:3 ratio upon reaching 60-80\% confluence, and these cells were cultured for a total of 6 generations in a $5 \% \mathrm{CO}_{2}$ incubator at $37^{\circ} \mathrm{C}$. Subsequent passages of the HCC-CR cells were referred to in a sequential numerical order starting at P1. HCC-CR cells were observed using an optical microscope (magnification, x10; Olympus Corporation, Tokyo, Japan) following culture for 50 and $100 \mathrm{~h}$.

Cell colony formation and viability assay. Following culturing under CR conditions for six passages, the cell colony formation per passage was observed using an inverted phase-contrast microscope (magnification, x10; Olympus Corporation). The HCC-CR cells were stained using a Live/Dead Molecular Probes staining kit (Invitrogen; Thermo Fisher Scientific, Inc.) for $30 \mathrm{~min}$ at $37^{\circ} \mathrm{C}$ and then analyzed using an inverted fluorescence microscope (magnification, x10; Olympus Corporation). Three images/sample were acquired for analysis. 
A

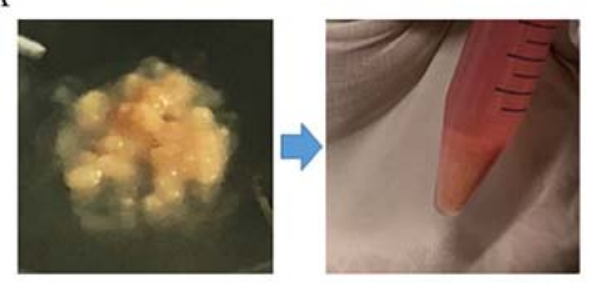

B

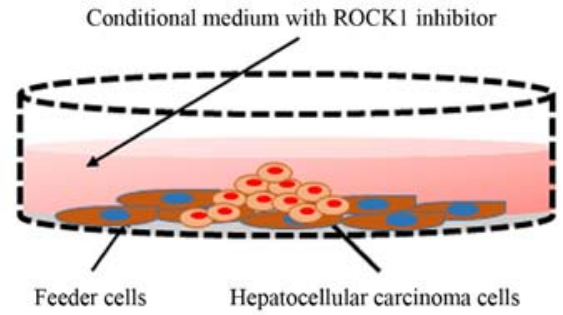

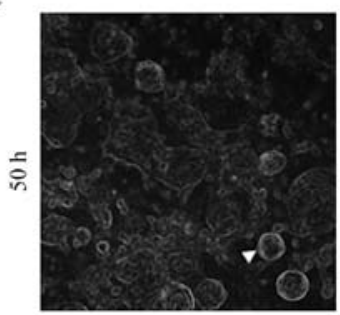

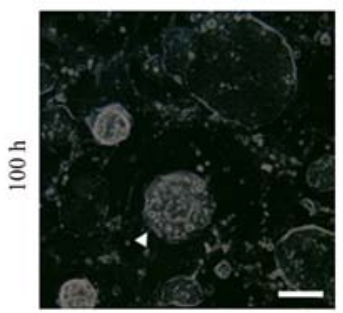

Figure 1. Isolation and CR culture of primary HCC cells. (A) Acquisition of HCC cells from tumor specimens. (B) Schematic diagram of CR culture. (C) HCC cell colonies following CR culture. Scale bar $=100 \mu \mathrm{m}$. CR, conditional reprogramming; HCC, hepatocellular carcinoma; ROCK1, rho-associated coiled-coil containing protein kinase 1 .

Cell proliferation assay. The proliferative ability of the $\mathrm{P} 0$ HCC-CR cells following culturing for 10 days was quantified via 5-ethynyl-2'-deoxyuridine (EdU)/Hoechst 33342 staining using a Cell-Light ${ }^{\mathrm{TM}}$ EdU in vitro imaging kit (Guangzhou RiboBio Co., Ltd., Guangzhou, China). Briefly, HCC-CR cells $\left(4 \times 10^{4}\right.$ cells $\left./ \mathrm{cm}^{2}\right)$ were seeded into a 24 -well plate and incubated with $50 \mathrm{mM}$ EdU labeling solution $(200 \mathrm{ml})$ at $37^{\circ} \mathrm{C}$ under $5 \% \mathrm{CO}_{2}$ for $3 \mathrm{~h}$. The HCC-CR cells were then sequentially treated with $4 \%$ paraformaldehyde (PFA; $\mathrm{pH} 7.4)$ for $30 \mathrm{~min}, 2 \mathrm{mg} / \mathrm{ml}$ glycine for $5 \mathrm{~min}, 0.5 \%$ Triton X-100 for $10 \mathrm{~min}$, anti-EdU working solution for $30 \mathrm{~min}$ and $5 \mathrm{mg} / \mathrm{ml}$ Hoechst 33342 dye for $30 \mathrm{~min}$ (all at room temperature). The cells were imaged under a fluorescence microscope (magnification, x10; Leica Microsystems GmbH, Wetzlar, Germany). Three images/sample were acquired for analysis. The numbers of HCC-CR cells were counted for each passage, and a plot of accumulated population doublings versus growth days was constructed following culturing for 10, 14, 22 and 30 days as previously described (19).

Western blotting. HCC-CR cells were separated from feeder cells by differential trypsinization. Briefly, the cells were washed by PBS, and then incubated by $0.05 \%$ trypsinization for $1 \mathrm{~min}$ at $37^{\circ} \mathrm{C}$ under $5 \% \mathrm{CO}_{2}$. The feeder cells were separated by tapping the bottom of the plates. Then, total protein was extracted from HCC-CR cells using lysis buffer (10 mM Tris, $150 \mathrm{mM} \mathrm{NaCl}$, $1 \%$ Triton X-100, $1 \%$ sodium deoxycholate, $0.1 \%$ SDS, $10 \mathrm{mM}$ EDTA and protease inhibitor cocktail, $\mathrm{pH}$ 7.4) on ice. The lysates were centrifuged at $14,000 \mathrm{x} \mathrm{g}$ for $10 \mathrm{~min}$ at $4^{\circ} \mathrm{C}$. The supernatants were then collected and the concentration of total protein was determined using a bicinchoninic acid assay kit (Beyotime Institute of Technology) according to the manufacturer's protocols. Equal amount of protein ( $30 \mu \mathrm{g} / \mathrm{lane})$ of the samples were boiled in water with SDS-PAGE sample loading buffer (Beyotime Institute of Technology) for $10 \mathrm{~min}$ prior to separation via $10 \%$ SDS-PAGE. The proteins were transferred onto polyvinylidene difluoride membranes (Roche Diagnostics, Basel, Switzerland) and blocked with 5\% non-fat milk at room temperature for $1 \mathrm{~h}$. Then the proteins. Then the membranes were incubated with primary antibodies against $\alpha$-fetoprotein (AFP; 1:1,000; cat. no. 4448; Cell Signaling Technology, Inc., Danvers, USA) and $\beta$-actin (1:1,000; cat. no. AF0003; Beyotime Institute of Technology). The membranes were then incubated with horseradish peroxide-conjugated secondary antibodies (1:1,000; cat. nos. A0208 and A0216; Beyotime Institute of Technology) for $1 \mathrm{~h}$ at room temperature and washed with TBS containing $1 \%$ Tween and PBS. Protein bands were visualized using the enhanced chemiluminescence (ECL) detection system (Pierce; Thermo Fisher Scientific, Inc.) with an ECL kit (EMD Millipore, Billerica, MA, USA). Protein expression was quantified using Quantity One 4.62 software (Bio-Rad Laboratories, Inc., Hercules, CA, USA).

Immunofluorescence staining. HCC-CR cells $\left(4 \times 10^{4}\right.$ cells $\left./ \mathrm{cm}^{2}\right)$ were seeded into a 24-well plate and incubated with $50 \mathrm{mM}$ EdU labeling solution $(200 \mathrm{ml})$ at $37^{\circ} \mathrm{C}$ under $5 \% \mathrm{CO}_{2}$ for $3 \mathrm{~h}$. Then the cells were fixed with $4 \%(\mathrm{v} / \mathrm{v})$ PFA for $10 \mathrm{~min}$ at room temperature, permeabilized with $0.3 \%$ Triton $\mathrm{X}-100$ and then blocked with $1 \%(\mathrm{w} / \mathrm{v})$ bovine serum albumin (cat. no. P0023B; Beyotime Institute of Technology) at $37^{\circ} \mathrm{C}$ for $30 \mathrm{~min}$. Following incubation overnight at $4^{\circ} \mathrm{C}$ with a primary antibody (mouse anti-human AFP; 1:200; cat. no. ab3980; Abcam, Cambridge, UK), the cells were washed with PBS and incubated with an Alexa Fluor ${ }^{\circledR} 488$-conjugated secondary antibody $(1: 1,000$; cat. no. R37120; Invitrogen; Thermo Fisher Scientific, Inc.) at room temperature for $1 \mathrm{~h}$. The cells were then incubated with anti-EdU working solution for $30 \mathrm{~min}$ and $100 \mathrm{ng} / \mathrm{ml}$ DAPI (Beyotime Institute of Technology, Shanghai, China) for $5 \mathrm{~min}$ (all at room temperature). and the samples were analyzed using a confocal laser scanning microscope (magnification, x10; SP5; Leica Microsystems GmbH, Wetzlar, Germany).

Statistical analysis. Data were analyzed using using SPSS software 22.0 (SPSS, Inc., Chicago, IL, USA). AFP protein expression data were analyzed by one-way analysis of variance, whereas cell proliferation data at different time points 


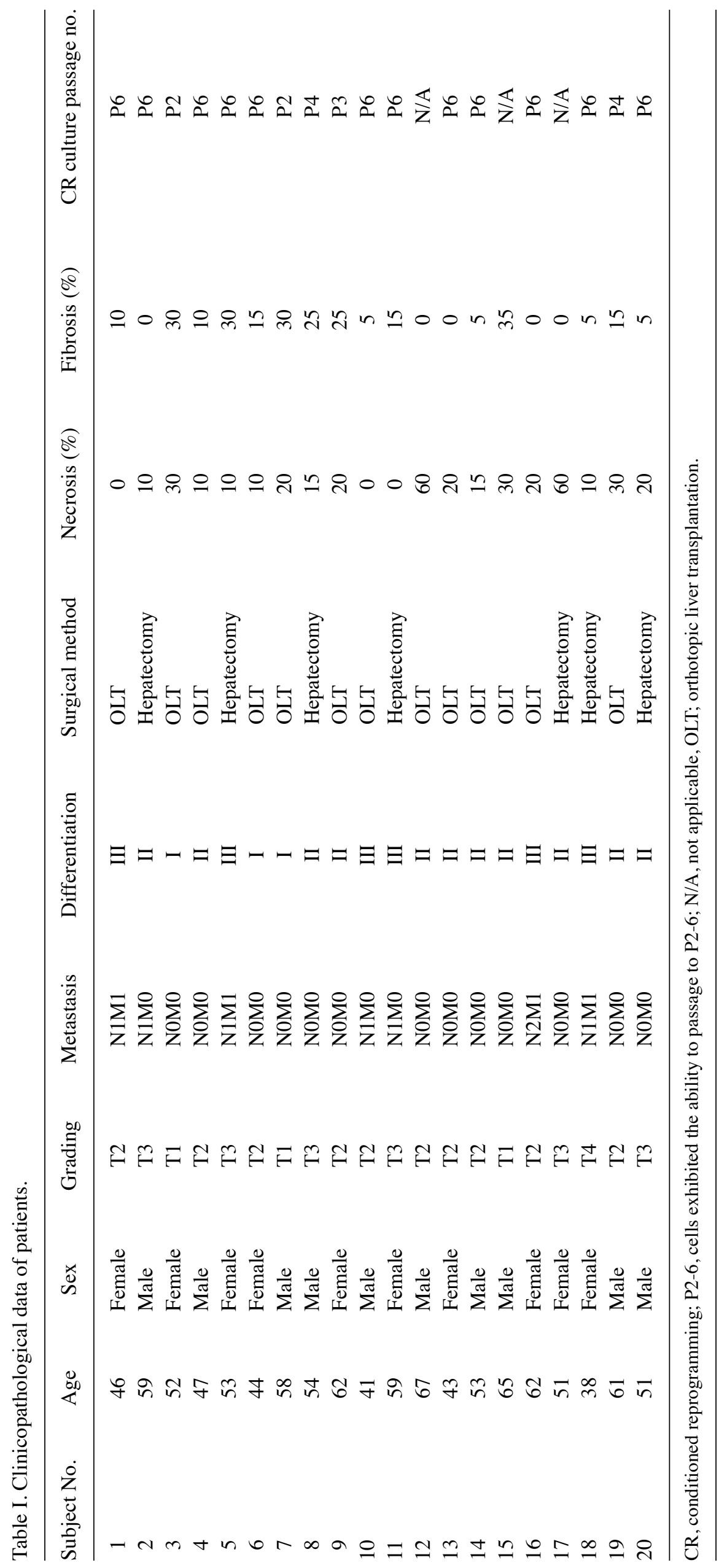


A
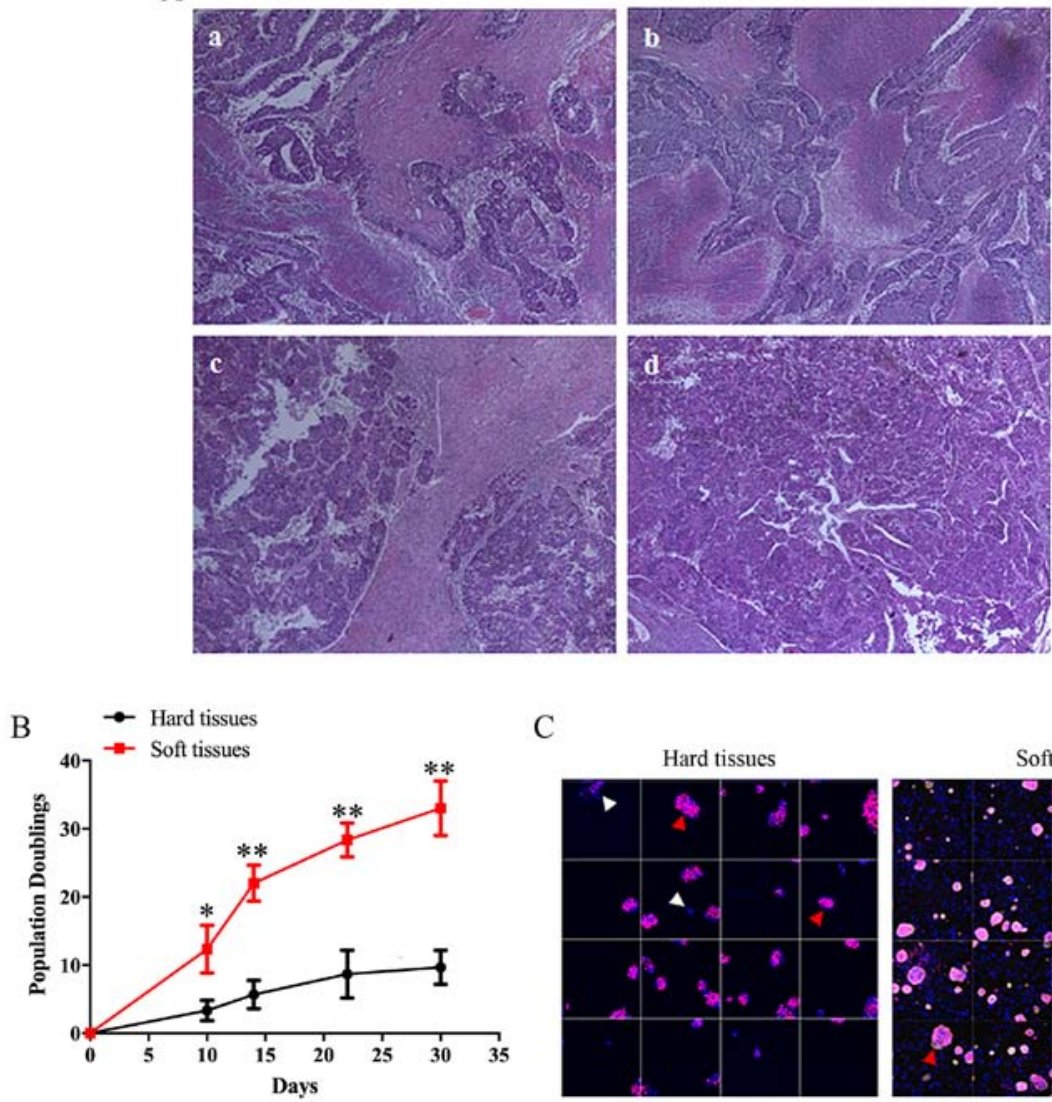

C
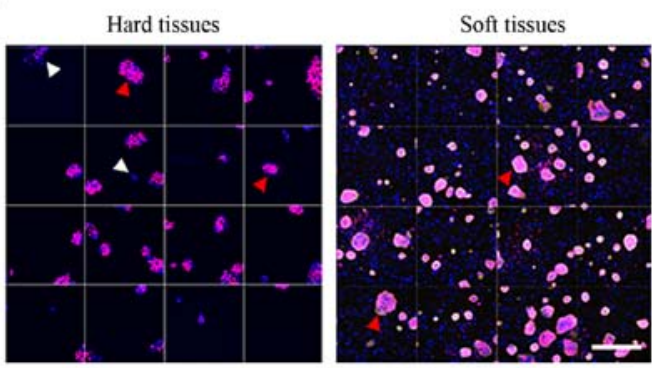

Figure 2. Proliferative ability of HCC-CR cells. (A) H\&E staining of tumor tissues (magnification, x40). A-a, II-differentiated tissue with $30 \%$ necrosis and $30 \%$ fibrosis; A-b, II-differentiated tissue with $60 \%$ necrosis; A-c, III-differentiated tissue with 35\% fibrosis and A-d, III-differentiated tissue with no necrosis and fibrosis. (B) Population doublings of HCC-CR cells isolated from hard and soft tissues were detected following culturing for 10, 14, 22 and 30 days. (C) 5-Ethynyl-2'-deoxyuridine (red) and Hoechst 33342 (blue) staining of HCC-CR cells following culture for 10 days. A notable increase in the number of colonies were observed from cells of soft tissue compared with hard tissue (white arrows); fewer proliferative cells were observed in hard tissues compared with soft tissues (red arrows). Scale bar $=300 \mu \mathrm{m}$. Data are presented as the mean \pm standard deviation. ${ }^{*} \mathrm{P}<0.05,{ }^{* *} \mathrm{P}<0.01$ vs. hard tissues. HCC-CR, conditioned reprogramming culture of hepatocellular carcinoma cells.

were analyzed using Student's t-tests. Data were presented as the mean \pm standard deviation of at least three independent experiments. Associations between the success rate of HCC-CR culture and patients' background, including gender and surgical method, were analyzed with $\chi^{2}$ tests. $\mathrm{P}<0.05$ was considered to indicate a statistically significant difference.

\section{Results}

Establishment of patient-derived primary HCC-CR cell cultures. Tumor tissue samples from 20 patients with HCC that had not been previously treated with radiotherapy or chemotherapy were collected, harvested and used for cell culture in the present study (Fig. 1A). Cell cultures were established by seeding HCC cells onto an NIH-3T3 fibroblast feeder layer in CR medium (Fig. 1B). As presented in Fig. 1C, adherent HCC cells exhibited small colonies at $50 \mathrm{~h}$ and progressed into cell islands by $100 \mathrm{~h}$.

The clinicopathological data of the patients are presented in Table I. The median age of the patients was 54.7 years; all patients suffered from HBV and/or HCV. Tissue samples were obtained during OLT or hepatectomy. A total of 35\% (7/20) of patients exhibited T3/T4 grade tumors. Additionally, 35\% (7/20) of patients had tumor metastasis. HCC cells from $55 \%$ of tumor tissues underwent successful continuous passaging under CR conditions; the ability to establish continuous passaging was markedly associated with the source and composition of tumor tissues. With the exception of tissues from $100 \%$ of T1 patients and $54.5 \%$ of T2 patients, HCC cells were obtained via CR culture from tissues from all other patients. HCC cells from metastatic patients were more likely to be expanded in vitro. The success rate of establishing $\mathrm{CR}$ cultures was independent of the surgical method used $(\mathrm{P}=0.9510)$. HCC-CR cells were not successfully generated from tissues with a necrosis rate $>30 \%$ and a stromal ratio $>35 \%$.

Propagation potential of primary HCC-CR cells. Complete hepatic nodule or blood vessel-rich tissues in primary HCC lesions were selected for further CR culture. A 6-day culture period was required to generate larger cell clones prior to the initial passage of $\mathrm{PO}$ cells, after which $\mathrm{HCC}$ cells were generated every 3-4 days. CR cultures were determined to possess the potential for continuous generation providing cells were generated on a 3-4-day cycle, and colonies demonstrated a continuous increase in quantity and volume. The morphologies of various tumor tissues were observed by H\&E staining (Fig. 2A). Harder tissues exhibited larger fibrotic areas and smaller necrotic regions. As presented in Fig. 2B, the 
A
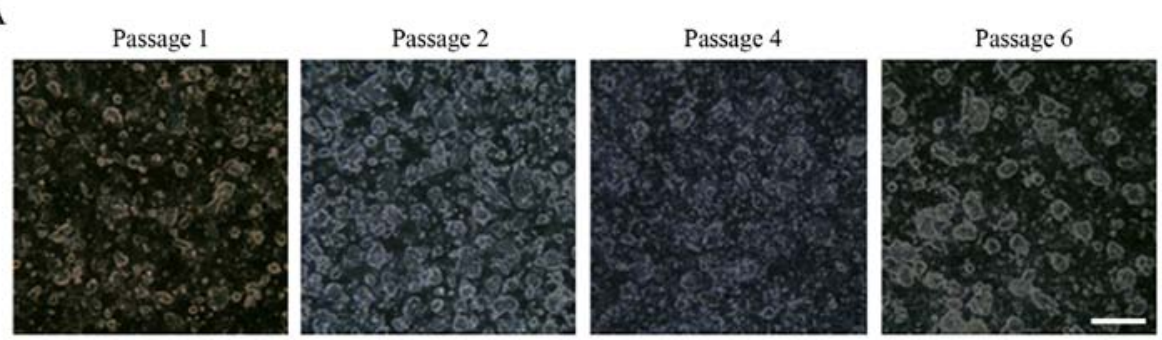

B
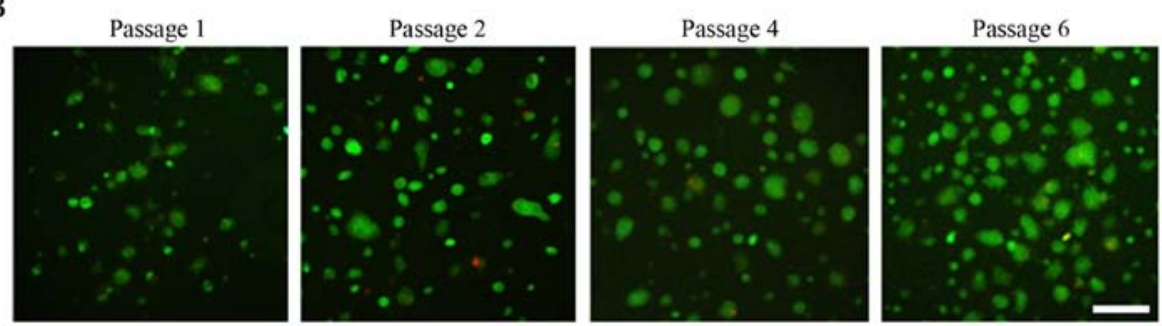

Figure 3. Continuous generation capacity of HCC-CR cells. (A) Cell colony formation per passage was observed using an inverted phase-contrast microscope. (B) Live (green) and dead (red) staining of HCC-CR cell colonies during Passage 1, 2, 4 and 6. Scale bar=100 $\mu \mathrm{m}$. HCC-CR, conditioned reprogramming culture of hepatocellular carcinoma cells.

A
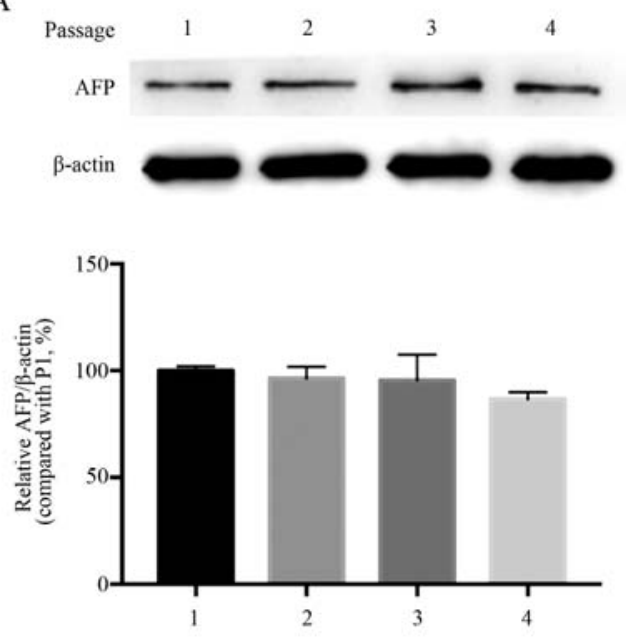

B
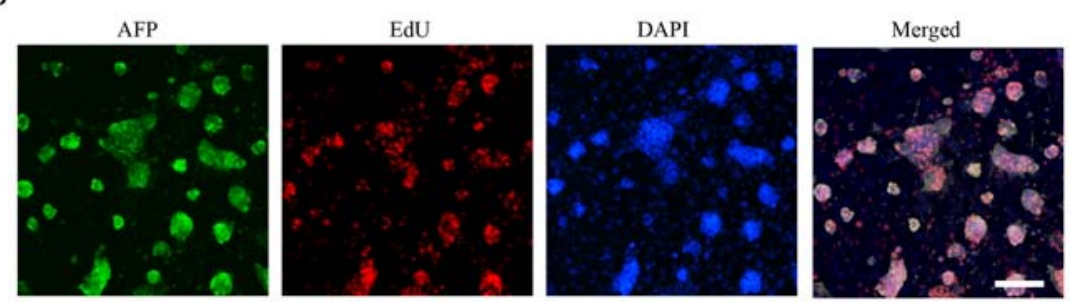

Figure 4. Tumor-specific marker expression of HCC-CR cells. (A) Expression of AFP in HCC-CR cells as determined via western blotting. (B) Staining of HCC-CR cells cultured for 40 days. AFP was stained by Alexa Fluor ${ }^{\circledR} 488$ (green), proliferative nuclei were stained by EdU (red) and nuclei were stained by DAPI (blue). Scale bar=100 $\mu \mathrm{m}$. AFP, $\alpha$-fetoprotein; EdU, 5-ethynyl-2'-deoxyuridine; HCC-CR, conditioned reprogramming culture of hepatocellular carcinoma cells.

proliferation of cells obtained from brittle, non-viscous tissues (hard tissues) during continuous cultivation was significantly decreased compared with cells from soft tissues with lumpy masses. Additionally, following culture for 6 days, a markedly increased number of colonies was observed in the soft tissues group compared with in the hard tissues group (Fig. 2C). Furthermore, EdU staining revealed that the number of cells with proliferative potential in hard tissues cell colonies was notably reduced compared with in soft tissue cell colonies.

Continuous generation capacity of HCC-CR cells. HCC-CR cells that possessed proliferative ability in the CR system were subjected to cryopreservation and resuscitation during every passage. As presented in Fig. 3A, HCC-CR cell colonies 
exhibited continuous increases in quantity and volume across generations. Additionally, live/dead cell staining revealed that HCC-CR cells retained high viability following repeated cryopreservation and resuscitation (Fig. 3B).

Expression of AFP in HCC-CR cells. The identity of the cultured cells was demonstrated by the stable expression of the HCC marker AFP during continuous generation (Fig. 4A). AFP expression was markedly downregulated with increasing passages; however, there was no significant difference. Immunofluorescence and EdU staining revealed that the cell purity was $>90 \%$ and the majority of AFP-positive cells possessed high proliferative potential (Fig. 4B).

\section{Discussion}

It was previously hypothesized that only a small proportion of tumor cells were able to form colonies in vitro (20). In particular, certain human epithelial cells, including those from the prostate, lung and liver, possess very short lifespans in vitro and can only undergo a limited number of passages, reducing their potential use in cell biology studies (9). A novel culture system has been developed for the indefinite propagation of various primary human cells (normal and tumor cells) in vitro via co-culture with irradiated fibroblast feeder cells and the rho-associated coiled-coil containing protein kinase 1 inhibitor Y-27632 (21). This culture system is termed CR due to the conditional induction of cell proliferation. As self-renewal is a reported property of stem cells, it was hypothesized that CR culture induced adult cells to exhibit adult stem cell-associated properties (22).

The aim of the present study was to establish a culture system in vitro for human hepatoma cells. Recent studies have reported the ability of cancer cells to exhibit sustained stable amplification under CR conditions (10-14). Numerous studies have made advancements in the field of liver cancer cell amplification; however, sufficient rapid amplification of cells was not established for clinical use (23). A previous study reported the successful construction of a primary HCC organoid; however, the expansion time required to generate the $3 \mathrm{D}$ organoid was impractically long (16). In the present study, HCC cells were passaged every 3-4 days. The numbers of harvested cells were counted following each passage, and a constant growth of HCC-CR cells during each passage was observed for 30 days (Fig. 2B). These findings were consistent with previous studies and meet clinical requirements $(14,24)$. The identification of additional biomarkers is required to further verify the reliability of the in vitro $\mathrm{CR}$ culture method in obtaining matched tumor cells from tissues from patients with HCC.

The ability to expand cells isolated from tissue samples in vitro was associated with the quality of the original tissue. In the present study, the HCC cells that were continuously passaged under CR conditions were obtained from $\sim 55 \%$ of tumor samples; this ability was affected by the origin and volume of actively proliferating tissue, as HCC cells from necrotic or fibrotic tissues struggled to be continuously passaged. As presented in Table I, the success rate of HCC-CR culture was independent of the age of patients and the surgical method used, which was consistent with a study investigating nasopharyngeal carcinoma (25). Additionally, the proliferation and cloning abilities of cells were notably increased in tissue samples from patients with metastasis compared with in other samples. Cells for amplification could not be isolated from calcified and necrotic lesions. Furthermore, reduced proliferation and activation was observed in tumor cells isolated from fibrotic samples. Based upon these findings, to obtain HCC-CR cells that can be used for subsequent experiments, original samples should be extracted from patients with tumors in the T2/T3 stage. Furthermore, combined with the sample information and the cell culture effect, it was suggested that the tumor tissue should be $>1 \mathrm{~cm}^{3}$ in size, with a necrosis rate of $<50 \%$ and a stromal ratio of $<40 \%$ to achieve acceptable expansion.

Increases in the number and area of cell colonies are regarded as manifestations of the proliferation of tumor cells in vitro (26). In the present study, successfully amplified HCC-CR cells formed more and larger cell colonies, thereby exhibiting adaptability to the CR medium. Furthermore, the proliferation of cells, formation of colonies and expression of AFP were markedly unaffected by repeated cycles of cryopreservation and resuscitation, indicating that the CR system met the requirements for successful hepatoma cell culture. On the contrary, due to the apparent complexity of the HCC-CR medium and the diversity of clinical samples, further investigation is required to develop a simpler, more versatile CR medium. Compared with tumor cells cultured under CR conditions, commercial tumor cell lines fail to fully reproduce important features of tumors in vivo. The heterogeneity of tumors results in susceptibility to drug resistance, leading to tumor recurrence and metastasis. In clinical settings, cells cultured under CR conditions may be useful for drug screening. In the present study, tumor cells were divided into soft and hard tissue groups. In addition, stable expression of the HCC marker AFP during continuous passaging was determined via western blotting. AFP is the most extensively studied serological biomarker in the surveillance of $\mathrm{HCC}$ and the only marker that has undergone all five phases of biomarker development $(27,28)$. The findings of the present study are promising; however, the CR system varies from physiological conditions. Therefore, analysis at the transcriptional and translation levels should be conducted, and in vivo transplants of CR-derived tumors should be performed.

In conclusion, 20 tumor specimens from patients with HCC were collected. HCC cells isolated from 55\% of samples exhibited continuous expansion under CR conditions; the ability to undergo continuous passaging was associated with the source and composition of the original tumor tissues. The expression of AFP in HCC-CR cells was stable during passaging, and cells demonstrated adaptability to CR culture conditions. These findings indicated that the CR system may be a useful source for passaging HCC cells required for clinical trials; however, the specific media components require further optimization.

\section{Acknowledgements}

Not applicable.

\section{Funding}

The present study was supported by the Tianjin Clinical Research Center for Organ Transplantation Project (grant no. 15ZXLCSY00070), the International S\&T Cooperation 
Program of China (grant no. 2015DFG31850), the Tianjin Science and Technology Plan Project (grant no. 14RCGFSY00147) and the National Human Genetic Resources Sharing Service Platform (grant no. 2005DKA21300).

\section{Availability of data and materials}

All data generated or analyzed during the present study are included in this published article.

\section{Authors' contributions}

ZW made substantial contributions to the conception and design of the present study, and the acquisition, analysis and interpretation of data. BB and HS performed the experiments. LL collected and analyzed patient information, and was involved in drafting the manuscript and revising it critically for important intellectual content. HZ performed some of the experiments and provided final approval for the present version to be published. SW was involved in the analysis and interpretation of data. ZS made substantial contributions to the design of the study and agreed to be accountable for all aspects of the study in ensuring that questions regarding the accuracy or integrity of any part of the work were appropriately investigated and resolved.

\section{Ethics approval and consent to participate}

The present study was approved by the Tianjin First Central Hospital Clinical Research Ethics Committee (review no. 2016N057KY).

\section{Patient consent for publication}

Not applicable.

\section{Competing interests}

The authors declare that they have no competing interests.

\section{References}

1. Bertuccio P, Turati F, Carioli G, Rodriguez T, La Vecchia C, Malvezzi M and Negri E: Global trends and predictions in hepatocellular carcinoma mortality. J Hepatol 67: 302-309, 2017.

2. Ghouri YA, Mian I and Rowe JH: Review of hepatocellular carcinoma: Epidemiology, etiology, and carcinogenesis. J Carcinog 16: 1, 2017.

3. Chan SL and Yeo W: Development of systemic therapy for hepatocellular carcinoma at 2013: Updates and insights. World J Gastroenterol 20: 3135-3145, 2014.

4. Tovoli F, Granito A, De Lorenzo S and Bolondi L: Regorafenib for the treatment of hepatocellular carcinoma. Drugs Today (Barc) 54: 5-13, 2018.

5. Thorgeirsson SS and Grisham JW: Molecular pathogenesis of human hepatocellular carcinoma. Nat Genet 31: 339-346, 2002.

6. Lohitesh K, Chowdhury R and Mukherjee S: Resistance a major hindrance to chemotherapy in hepatocellular carcinoma: An insight. Cancer Cell Int 18: 44, 2018.

7. Huch M, Gehart H, van Boxtel R, Hamer K, Blokzijl F, Verstegen MM, Ellis E, van Wenum M, Fuchs SA, de Ligt J, et al: Long-term culture of genome-stable bipotent stem cells from adult human liver. Cell 160: 299-312, 2015.

8. Liu X, Ory V, Chapman S, Yuan H, Albanese C, Kallakury B, Timofeeva OA, Nealon C, Dakic A, Simic V, et al: ROCK inhibitor and feeder cells induce the conditional reprogramming of epithelial cells. Am J Pathol 180: 599-607, 2012.
9. Liu X, Krawczyk E, Suprynowicz FA, Palechor-Ceron N, Yuan H, Dakic A, Simic V, Zheng YL, Sripadhan P, Chen C, et al: Conditional reprogramming and long-term expansion of normal and tumor cells from human biospecimens. Nat Protoc 12: 439-451, 2017.

10. Mahajan AS, Sugita BM, Duttargi AN, Saenz F, Krawczyk E, McCutcheon JN, Fonseca AS, Kallakury B, Pohlmann P, Gusev Y, et al: Genomic comparison of early-passage conditionally reprogrammed breast cancer cells to their corresponding primary tumors. PLoS One 12: e0186190, 2017.

11. Alamri AM, Kang K, Groeneveld S, Wang W, Zhong X, Kallakury B, Hennighausen L, Liu X and Furth PA: Primary cancer cell culture: Mammary-optimized vs conditional reprogramming. Endocr Relat Cancer 23: 535-554, 2016.

12. Gao B, Huang C, Kernstine K, Pelekanou V, Kluger Y, Jiang T, Peters-Hall JR, Coquelin M, Girard L, Zhang W, et al: Non-malignant respiratory epithelial cells preferentially proliferate from resected non-small cell lung cancer specimens cultured under conditionally reprogrammed conditions. Oncotarget 8: 11114-11126, 2017.

13. Saeed K, Rahkama V, Eldfors S, Bychkov D, Mpindi JP, Yadav B, Paavolainen L, Aittokallio T, Heckman C, Wennerberg K, et al: Comprehensive Drug Testing of Patient-derived Conditionally Reprogrammed Cells from Castration-resistant Prostate Cancer. Eur Urol 71: 319-327, 2017.

14. Timofeeva OA, Palechor-Ceron N, Li G, Yuan H, Krawczyk E, Zhong X, Liu G, Upadhyay G, Dakic A, Yu S, et al: Conditionally reprogrammed normal and primary tumor prostate epithelial cells: A novel patient-derived cell model for studies of human prostate cancer. Oncotarget 8: 22741-22758, 2017.

15. Sette G, Salvati V, Giordani I, Pilozzi E, Quacquarini D, Duranti E, De Nicola F, Pallocca M, Fanciulli M, Falchi M, et al: Conditionally reprogrammed cells (CRC) methodology does not allow the in vitro expansion of patient-derived primary and metastatic lung cancer cells. Int J Cancer 143: 88-99, 2018.

16. Broutier L, Mastrogiovanni G, Verstegen MM, Francies HE, Gavarró LM,Bradshaw CR, Allen GE, Arnes-Benito R, Sidorova O, Gaspersz MP, et al: Human primary liver cancer-derived organoid cultures for disease modeling and drug screening. Nat Med 23: 1424-1435, 2017.

17. World Medical A; World Medical Association: World Medical Association Declaration of Helsinki: Ethical principles for medical research involving human subjects. JAMA 310: 2191-2194, 2013.

18. Thery C, Amigorena S, Raposo G and Clayton A: Isolation and characterization of exosomes from cell culture supernatants and biological fluids. Curr Protoc Cell Biol 30: 3.22.1-3.22.29, 2006.

19. Zhu Y, Yang Y, Guo J, Dai Y, Ye L, Qiu J, Zeng Z, Wu X, Xing Y, Long X, et al: Ex vivo 2D and 3D HSV-2 infection model using human normal vaginal epithelial cells. Oncotarget 8: 15267-15282, 2017.

20. Plaks V, Kong N and Werb Z: The cancer stem cell niche: How essential is the niche in regulating stemness of tumor cells? Cell Stem Cell 16: 225-238, 2015.

21. Agarwal S and Rimm DL: Making every cell like HeLa a giant step for cell culture. Am J Pathol 180: 443-445, 2012.

22. Suprynowicz FA, Upadhyay G, Krawczyk E, Kramer SC, Hebert JD, Liu X, Yuan H, Cheluvaraju C, Clapp PW, Boucher RC Jr, et al: Conditionally reprogrammed cells represent a stem-like state of adult epithelial cells. Proc Natl Acad Sci USA 109: 20035-20040, 2012.

23. Gillet JP, Varma S and Gottesman MM: The clinical relevance of cancer cell lines. J Natl Cancer Inst 105: 452-458, 2013.

24. Hensley CT, Wasti AT and DeBerardinis RJ: Glutamine and cancer: Cell biology, physiology, and clinical opportunities. J Clin Invest 123: 3678-3684, 2013

25. Yu F, Lu Y, Tao L, Jiang YY, Lin DC, Wang L, Petersson F, Yoshiyama H, Koeffler PH, Goh BC, et al: Non-malignant epithelial cells preferentially proliferate from nasopharyngeal carcinoma biopsy cultured under conditionally reprogrammed conditions. Sci Rep 7: 17359, 2017.

26. Reya T, Morrison SJ, Clarke MF and Weissman IL: Stem cells, cancer, and cancer stem cells. Nature 414: 105-111, 2001.

27. McMahon BJ, Alberts SR, Wainwright RB, Bulkow L and Lanier AP: Hepatitis B-related sequelae. Prospective study in 1400 hepatitis B surface antigen-positive Alaska native carriers. Arch Intern Med 150: 1051-1054, 1990.

28. Zhang BH, Yang BH and Tang ZY: Randomized controlled trial of screening for hepatocellular carcinoma. J Cancer Res Clin Oncol 130: 417-422, 2004.

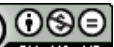

This work is licensed under a Creative Commons Attribution-NonCommercial-NoDerivatives 4.0 International (CC BY-NC-ND 4.0) License. 